\title{
Gabriela Signori, Birgit Studt (dir.), Das Konstanzer Konzil als europaïsches Ereignis. Begegnungen, Medien und Rituale
}

Ludovic Viallet

\section{OpenEdition}

Édition électronique

URL : http://journals.openedition.org/ifha/8674

DOI : $10.4000 /$ ifha. 8674

ISSN : 2198-8943

\section{Éditeur}

IFRA - Institut franco-allemand (sciences historiques et sociales)

Référence électronique

Ludovic Viallet, « Gabriela Signori, Birgit Studt (dir.), Das Konstanzer Konzil als europaïsches Ereignis. Begegnungen, Medien und Rituale ", Revue de l'IFHA [En ligne], Date de recension, mis en ligne le 26 janvier 2017, consulté le 24 septembre 2020. URL : http://journals.openedition.org/ifha/8674 ; DOI : https://doi.org/10.4000/ifha.8674

Ce document a été généré automatiquement le 24 septembre 2020.

(CIFHA 


\section{Gabriela Signori, Birgit Studt (dir.), Das Konstanzer Konzil als europaïsches Ereignis. Begegnungen, Medien und Rituale}

Ludovic Viallet

\section{RÉFÉRENCE}

Gabriela Signori, Birgit Studt (dir.), Das Konstanzer Konzil als europaïsches Ereignis. Begegnungen, Medien und Rituale, Ostfildern: Jan Thorbecke (Vorträge und Forschungen herausgegeben vom Konstanzer Arbeitskreis für mittelalterliche Geschichte, 79), 2014, 416 p., $58 €$ 
Étant donné la masse de la production historiographique consacrée aux conciles $\mathrm{du} \mathrm{Xv}^{\mathrm{e}}$ siècle, a fortiori en des années anniversaires, il n'allait pas de soi que cet ouvrage, issu en grande partie d'une

Das Konstanzer Konzil als europäisches Ereignis rencontre organisée à l'automne 2011 par les médiévistes de l'Université de Constance, trouve pleinement sa place. Le pari a toutefois été relevé dans la mesure où il envisage l'histoire du concile des bords $\mathrm{du}$ Bodensee sous un angle résolument européen, celui des répercussions de l'événement sur les entités nationales en construction - ontils agi, dans les différents lieux, comme une "force marquante ", selon les mots de Birgit Studt en clôture de l'ouvrage ?et celui des influences que les différents intérêts de ces dernières exercèrent sur son déroulement.

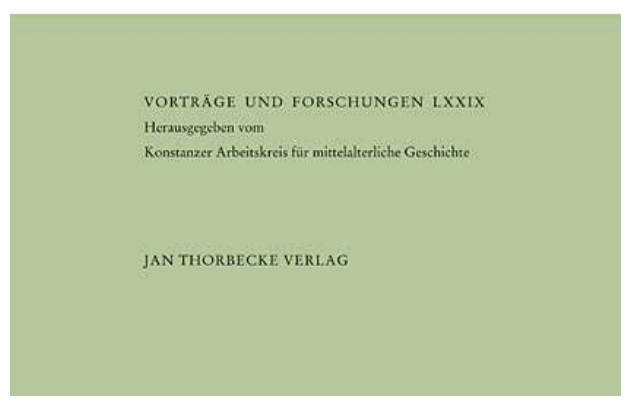

En consacrant son propos introductif à une stimulante réflexion sur la question des nationes, Gabriela Signori place d'emblée le lecteur dans cette perspective. On peut d'ailleurs parler, souligne B. Studt, non seulement d'une " fragmentation européenne " mais aussi d'un "décentrement ", comme le montre le cas de la nation ibérique, pour laquelle les choses se sont aussi jouées à Narbonne et à Perpignan en 1415. La contribution d'Ansgar Frenken aborde la Castille, celle de Nikolas Jaspert, l'Aragon en prenant comme point de départ le témoignage du bouffon catalan Antoni Tallander (alias Mossen Borra), qui séjourna à Constance puis en Europe centrale. Pour ces deux royaumes, l'élection de Martin $\mathrm{V}$ ouvrit une nouvelle ère dans les relations entre l'Église et l'État. Leur adhésion au concile avait entrâné des tensions relatives au système du vote par nation; derrière cette question se posait celle de la nationalité et de l'identité nationale au Moyen Âge, souligne Robert N. Swanson en reprenant à nouveaux frais la réponse du doyen d'York Thomas Polton, le 31 mars 1417, aux arguments français en faveur de l'intégration des Anglais dans la nation germanique. Pour l'Europe centrale, Pavel Soukup analyse les prédications des Bohémiens Étienne von Páleč, Matthieu von Königsaal et Maurice Rvačka, adversaires de Hus dès avant le concile, au cours duquel ils se mêlèrent aussi de réforme de l'Église. Constance a marqué une coupure dans la carrière d'Étienne et de Maurice, qui partirent en exil en Pologne, tandis que Matthieu (l'un des rares cisterciens à prêcher au concile) reprit sa vie claustrale. Si le cas bohémien présente évidemment de fortes spécificités, on y retrouve la même équation essentielle entre un global event, des parcours individuels et un arrière-pays - le contexte de l'espace d'origine des participants - qui fut tout sauf un arrière-plan. En témoigne la diplomatie des ambassades du roi de France à Constance, dont le bilan mitigé est dressé par Sophie Vallery-Radot, la signature du concordat par la nation française le 15 avril 1418 constituant une véritable rupture avec le gouvernement armagnac. 
D'autres contributions de l'ouvrage ont valeur de mises au point générales, dans lesquelles on puisera d'importants éléments d'information et de réflexion. On pense en particulier au texte de Johannes Helmrath sur ces décennies si particulières de la première moitié $\mathrm{du} \mathrm{xv}^{\mathrm{e}}$ siècle où se multiplièrent les assemblées en une véritable " synodalisation ", et à celui de Martin Kintzinger, qui, débutant par une (re-)lecture de l'historiographie centrée sur l'œuvre majeure de Walter Brandmüller, s'arrête notamment sur la notion de communication politique. À cet égard, Gerrit Jasper Schenk pose longuement la question de la lisibilité des signes du pouvoir et des limites du pouvoir des signes à partir du cas de l'entrée du pape pisan Jean xxiII dans Constance le 28 octobre 1414, dont il reconstitue, schémas à l'appui, le cortège processionnel afin de livrer une analyse de l'événement fondée notamment sur la Chronique d'Ulrich Richental. C'est aux listes de noms et de participants au concile figurant dans cette dernière que Thomas Martin Buck consacre son étude : une thématique de recherche en soi, avec ses problèmes de méthode et de traitement des sources (un répertoire des listes contenues dans les différents manuscrits est d'ailleurs donné en annexe). On appréciera ainsi que les analyses de fond ne s'effacent pas derrière la seule invocation de concepts - celui de Medialität par exemple. Enfin, deux contributions rappellent que Constance a aussi représenté un creuset culturel en offrant des possibilités exceptionnelles aux lettrés (les ambiguïtés du terme d'"humanistes" ne sont pas levées par Concetta Bianca) de se procurer et de copier des ouvrages, mais aussi en œuvrant à l'«européisation» de la culture musicale, comme le montre Therese Bruggisser-Lanker, dont l'étude est l'occasion d'évoquer le parcours d'hommes aussi différents que le chevalier Oswald von Wolkenstein, le chancelier parisien Jean Gerson ou l'évêque de Florence Francesco Zabarella.

Avec quelle encre doit-on désormais écrire l'histoire du concile de Constance? La question, posée par B. Studt, appelle à la multiplication des perspectives, au décloisonnement et à la mise en relation, au-delà des mots et des effets de mode auxquels les artisans de ce volume n'ont pas cédé.

\section{INDEX}

Index chronologique : Moyen Âge

Thèmes : Histoire des États et des pouvoirs, Histoire religieuse

\section{AUTEURS}

\section{LUDOVIC VIALLET}

Université Clermont-Ferrand 2 